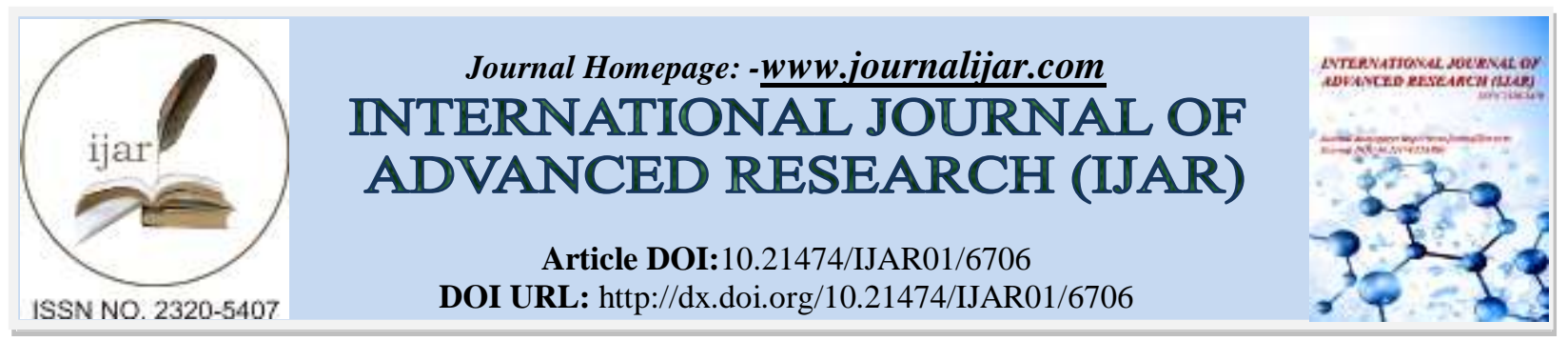

RESEARCH ARTICLE

\title{
RÉTINOPATHIE DE VALSALVA ET GROSSESSE.
}

\begin{abstract}
Ahmed. Meklaa ${ }^{1}$, Mounsef. Mahaouchi ${ }^{1}$, Omar. laghzaoui ${ }^{1}$ and Adil.Bouzidi ${ }^{2}$.
1. Service de gynécologie-obstétrique, hôpital militaire moulay ismail Meknes.

2. Service d'hophtalmologie, hôpital militaire moulay ismail Meknes.
\end{abstract}

\section{Manuscript Info}

Manuscript History

Received: 09 January 2018

Final Accepted: 11 February 2018

Published: March 2018

\section{Abstract}

Copy Right, IJAR, 2018,. All rights reserved.

\section{Introduction:-}

La rétinopathie hémorragique de Valsalva est une pathologie rare, souvent responsable d'une chute brutale de l'acuité visuelle associée à une hémorragie rétinienne pré-maculaire. La grossesse est considérée comme facteur de risque. Nous rapportons un cas de rétinopathie de Valsalva et grossesse, à travers une revue de la littérature nous décrivons le mécanisme physiopathologique de cette affection ainsi que les modalités de prise en charge thérapeutiques.

\section{Cas Clinique:-}

Patiente de 35 ans, enceinte de 16SA, admise aux urgences ophtalmologique pour baisse brutale de l'acuité visuelle de l'œil gauche. L'interrogatoire retrouve : une grossesse bien suivie ; vomissement gravidique à 09SA pour lequel elle a été hospitalisée dans notre formation pendant dix jours avec bonne évolution clinique sous traitement symptomatique ; un syndrome grippal avec toux les cinq derniers jours précédant son admission.

L'examen au fond d'œil révèle la présence d'une hémorragie pré-maculaire retro-hyaloïdienne(figure1). L'angiographie en fluorescence ne montre pas d'anomalie vasculaire particulière. Un bilan général a été réalisé notamment une NFS, une glycémie et un bilan d'hémostase qui sont revenus normaux. Le diagnostic de rétinopathie hémorragique de Valsalva est retenu.

La patiente a bénéficiée d'un traitement par laser sur la membrane hyaloidienne afin de disperser l'hématome permettant ainsi sa résorption.

\section{Discussion:-}

La rétinopathie de Valsalva a été décrite pour la première fois par Duane à Philadelphie en 1972 [1].

Le mécanisme physiopathologique des hémorragies rétrohyalö̈diennes secondaires à la manœuvre de Valsalva est connu : il existe des adhérences physiologiques du vitré aux vaisseaux rétiniens. L'hyperpression thoracique par blocage en inspiration se répercute sur la circulation veineuse céphalique et donc rétinienne. Sous l'effet de l'hyperpression veineuse rétinienne, les adhérences tirent le vaisseau qui se rompt dans la cavité vitréenne ou dans l'espace rétrohyaloïdien. C'est donc la traction vitréenne sur une veine, qui entraîne sa rupture et l'inondation sanguine [2]. 
L'origine d'hémorragie rétrohyalö̈dienne, peut survenir de façon très différente : pendant des efforts volontaires de vomissement, au cours des efforts de poussée précédant l'accouchement, ou pendant une activité sexuelle intense .... [3]. Pendant la grossesse, il existe déjà une augmentation de la pression intra-abdominale. Avec les efforts de vomissements et de toux, l' hyperpression céphalique devient considérable pour provoquer la rupture des capillaires rétiniens superficiels [4]. La grossesse est connue comme facteur de risque de cette affection. Le pronostic est en général bon avec une récupération spontanée dans la grande majorité des cas [4][5]. Le moment de la résorption spontanée est très variable. L'utilisation du laser YAG peut être proposée dans certains cas pour disperser les hémorragies prérétiniennes dans le vitré et permettre ainsi une récupération plus rapide [6].

\section{Conclusion:-}

La grossesse est un facteur de risque de rétinopathie de Valsalva. Le diagnostic doit être retenu après avoir éliminé les autres causes d'hémorragies. L'évolution est généralement spontanément favorable.

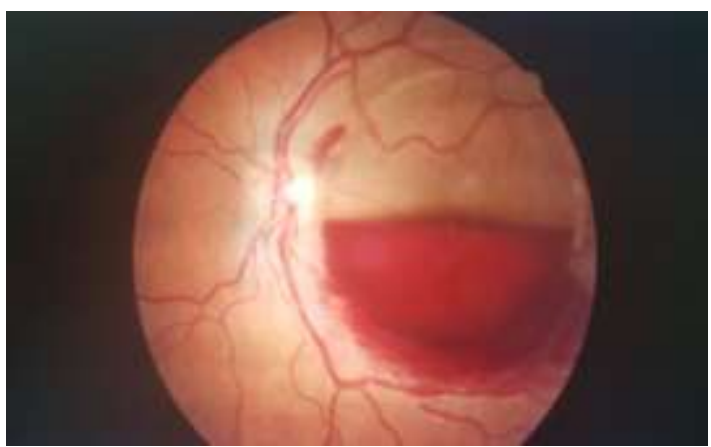

Figure 1:-Hemorragie pré-maculaire retro-hyaloidienne

\section{References:-}

1. Duane, T.D. (1972) Valsalva Hemorrhagic Retinopathy. Transactions of the AmericanOphthalmological Society, 70, 298-313.

2. Guigon-Souquet, B., Salaun, L., Macarez, R., Bazin, S., De La Manierre, E. andMazdou, M. (2004) Hemorragie rétrohyaloïdienne secondaireà une manoeuvre de Valsalva. Journal Français d'Ophtalmologie, 27, 1159-1162.

3. El-Khayat, A.R. (2017) Valsalva Haemorrhagic Retinopathy in Pregnancy after Yoga.BMJ Case Reports, 11.

4. Nghiem-Buffet, S. and Guiberteau, B. (2009) Rétinopathie de Valsalva et grossesse. Images en Ophtalmologie, 3, 86-87.

5. García-Fernández, M., Castro-Navarro, J. and Gonzalez-Castano, C. (2012) Long-Term Evolution of Valsalva Retinopathy: A Case Series. Journal of Medical Case Reports, 6, 346.

6. Matonti, F., Donadieu, V., Hoffart, L., Dornadin, A., Nadeau, S., Roux, S. andDenis, D. (2013) Traitement précoce par laser Nd:YAG de la rétinopathie de Valsalva: Résultants à propos de cinq cas et revue de la littérature. Journal Français d'Ophtalmologie, 36, 604-609. 